Antragsstellers unterzogen. Über diesen Schritt wird für den Domain-Inhaber und -Besucher noch mehr Sicherheit erreicht. Mit der Erweiterung der Comodo Prüfungsrichtlinien werden die vom Certificate Authority and Browser Forum (CA/B-Forum) verabschiedeten Anforderungen für die Ausgabe und das Management von Zertifikaten (http://www.cabforum.org/Baseline_Requirements_V1.pdf) erfüllt, die für alle führenden Zertifikatherausgeber und Browserhersteller ab dem 01. Juli bindend sind und mehr Sicherheit in der Webkommunikation gewährleisten sollen.

\section{Bundesdruckerei: Bereits eine Million elektronischer Aufenthaltstitel}

Elf Monate nach Einführung des elektronischen Aufenthaltstitels (eAT) im September 2011 besitzen bereits rund eine Million ausländische Mitbürger das neue ID-Dokument.

Seit dem 1. September 2011 erhalten Ausländerinnen und Ausländer in Deutschland den neuen elektronischen Aufenthaltstitel. Das ID-Dokument im Scheckkartenformat löst das bisher übliche Klebeetikett im Reisepass ab. Ziel ist es, die Aufenthaltstitel von Drittstaatsangehörigen, also von Ausländern, die nicht Staatsbürger der Europäischen Union sind, europaweit zu vereinheitlichen.

Die ID-Karte bietet ihren Inhabern die gleichen Möglichkeiten wie deutschen Bürgern der neue deutsche Personalausweis. Die Online-Ausweisfunktion ermöglicht es, Behördengänge online zu erledigen oder beim Online-Shopping seine Identität zu bestätigen. Voraussetzung dafür ist, dass die Dokumenteninhaber die Funktion bei Erhalt des Aufenthaltstitels nicht ausschalten lassen. Die Anbieter, die diesen Dienst in ihr Internetangebot integrieren, müssen ebenfalls ihre Identität eindeutig nachweisen. Ebenso wie der Personalausweis kann auch der Aufenthaltstitel zusätzlich für die Qualifizierte Elektronische Signatur (QES) genutzt werden. Sie ermöglicht es Internetnutzern zum Beispiel, rechtsverbindliche Verträge online zu unterschreiben.

Auf dem Chip im Inneren der Karte sind alle Daten gespeichert, die auf dem Aufenthaltstitel sichtbar sind, unter anderem das di- gitale biometrische Passfoto. Außerdem enthält er so genannte Nebenbestimmungen, wie zum Beispiel Angaben zur Erwerbstätigkeit und verpflichtend zwei Fingerabdrücke des Inhabers, sofern dieser älter ist als sechs Jahre. Sowohl auf die biometrischen Daten als auch auf die Nebenbestimmungen können nur hoheitliche Stellen wie Polizei, Grenzschutz oder Zoll zugreifen.

Seit der Einführung des neuen elektronischen Aufenthaltstitels stattet die Bundesdruckerei nicht nur die rund 5.400 Meldebehörden mit Änderungs- und Leseterminals sowie Software zur Beantragung eines neuen Personalausweises aus, sondern auch die rund 600 Ausländerbehörden, die den elektronischen Aufenthaltstitel ausstellen. Um die neuen Online-Funktionen der Ausweisdokumente nutzen zu können, stellt die Bundesdruckerei Berechtigungszertifikate und einen eigenen elD-Service für Online-Diensteanbieter bereit.

\section{IT Security Industrial \& Automation 2012 am 13. / 14.11.2012 in Leipzig}

Die IT Security Industrial \& Automation 2012 am 13. und 14.11.2012 in Leipzig ist die erste Konferenz der isits AG zum Thema Absicherung von Produktion und Automation, welche in Kooperation mit ESCRYPT GmbH und TÜV Rheinland stattfindet. Im Vordergrund stehen die Standards, die Analyse der Bedrohungslage, Angriffsszenarien, Unternehmensberichte und Absicherungsmöglichkeiten.

Deutschland ist einer der Innnovationsweltmeister. Dieses Wissen ist weltweit gefragt und dadurch werden deutsche Unternehmen zunehmend das Ziel von illegalen Attacken.

Welche Gefahren gibt es überhaupt? Wie komplex ist das Gesamtsystem? Wie viel Absicherung ist sinnvoll? Wie verhält sich eine Verschlüsselungslösung in einer komplexen Systemstruktur? Welche Möglichkeiten kann es geben, die Systemperformance beizubehalten?

Weitere Informationen: http://www.itsec-process.info/

sche Studien zur Auswirkung der Videoüberwachung dargestellt, wobei der Schwerpunkt auf der Kritik an den Studien und dem Bezweifeln ihrer (in der Tat widersprüchlichen) Ergebnisse liegt. Dieser Teil hätte auch gut bei der Frage der Geeignetheit der Videoüberwachung zur Erreichung der Kriminalprävention im Rahmen der Verhältnismäßigkeitsprüfung angesiedelt werden können. Dort (S. 139 - 142) wird aber lediglich auf die Darstellung der Studien am Anfang der Arbeit verwiesen.

Nach 66 Seiten beginnt dann die „rechtliche Bewertung" von Videoüberwachungsmaßnahmen, die lobenswerter Weise im Wesentlichen an das Prüfungsschema für die Rechtmäßigkeit einer polizeilichen Maßnahme angelehnt ist. Maximini stellt das Recht der informationellen Selbstbestimmung dar und auch die anderen Grundrechte (die Meinungs-, Versammlungs- und Religionsfreiheit, sowie die Unverletzlichkeit 